dans un patrimoine homozigrote, il faut aussi que celui-ci soit adéquat au milien dans lequel il doit se développer.

L'enpirisme sélectionniste a du bon, ct il y a eu de grands éleveurs avant la décourerte des lois de Vendel ; mais tout de mème, on va plus vite et phus loin lorsquion compreud ce que l'on fait. Exemple : depuis une dizaine d'années, des éleveurs soceupent du Lapin dit Castorrex dont la fontrure est particulièrement estimée, mais la transmission du caractère itait parfois imparfaite et quelques résultats paraissaient illogiques, puisquion a vu des Castorrex considérés comme parfaitement homozygotes donuer par leur croisement uniquement des Lapins normaux. Il a suffi à un généticicn de réfléchir sur les documents d'élevage qui étaient à sa disposition, pour établir la composition factorielle des Castorrex, de façon à re que l'on puisse opérer désormais en toute sécurité ct prévoir aver certitude ce que donnera tel ou tel croisement. Et on pourrait citer bien d'autres remples analogues.

\title{
CARPIGULTURE ÉLÉMENTAIRE
}

\section{Par le Conte de NEUfBourG}

De nombreus lecteurs du Bulletin demandent la publimation de notions simples, facilement assimilables par les noweaux venus à la mise en valeur de leurs étangs. Ils désirent surtout que res notions soient applirables, pratiques, et rendent des services se traduisant par un gain.

11 ne sagit donc pas d'expériences biologipues, d'observations de laboratoire, de compilation d'auteurs savants ou très décorés II faut que les vieux fleveurs, qui ont fait leurs écoles au dépens de leur poche, évitent aur jeunes des erreurs, des tâtonnements, des pertes d'argent ef de temps.

Iussi bien n y a-t-il pas d'enseisnement officiel pour l'étang : et cela vaut mieux, car cette culture ne sapprend bien que sanctionnée par l'intérèt pécunier du propriétaire exploitant. Je dis " propriétaire ", et non pas "fermier" : la conservation du capial foncier, chansées, bondes, fossés ; l’élevage mème de certains poissons, n̈intéressent pas an mème titre l'exploilant qui aflerme et celui qui possède.

Certes, l'Union des Syndicals de i'Flang a déjà mis an monde plusieurs

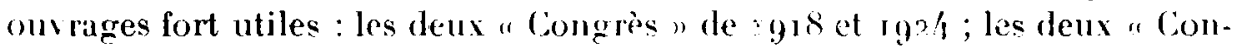

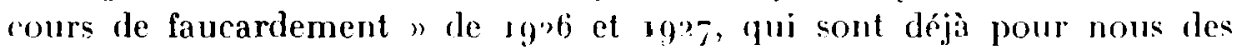
soụenirs de jeunesse, de camaraderie, d'études tenaces mais joveuses! Quant à la collection du Bulietin, qui comporte plus de 6o numéros, il est indispensable de la parcourir : votre Sundicat la possède complète. Outre de nombreuses notes sur la constitution des étangs, le matériel, l'alevinagre. la sélection, le faucardaze, les engrais, les maladies, les mours du Poisson of de ses ennemis, les viviers, le transport, la vente, Ies recettes, 
la jurisprudence, la fiscalité, les douanes, les expositions, on y trouve une dizaine de récits relatifs aux visites faites à des exploitations de types différents qui sont ae bonnes leçons de choses.

Ces récils confirment notre opinion personnclle, fruit d une expérience de quatre ou cinq lustres: il ne pent y avoir de bon traité général de pisriculture parce que chaque étang a sa nature propre, des conditions d'exposition, de sol, d'étendue, de forme, d'alimentation d'cau, de climat qui lui sont particulières.

L'expérience prouve que des f́tangs voisins, d'apparence analogue, réagissent différcmment à un même traitement.

Joignez-y que les conditions de vente sont très variables, selon les régions, et vous saurez que les plus savants ouvragres ne valent rien sils ne sont étudiés à la lumière d'une pratique locale d'au moins trois saisons de pèche.

Il est eruel, mais nécessaire, d'onlever ainsi dès l'abond une illusion aus néophytes qui espèrent trouver an quelques pages la loi et les prophètes de leur nouvelle occupation.

Si la lecture la plus assidue des manuels de la Baronne Srark n a jamais pu, seule, faire une lemme du monde, ni mème y contribuer beatucoup ; si la récitation exacte du vade-mecum de lapprenti basse-rourier ne suffit pas à faire gagner tout de suite quatre sous à l'ingénu qui poulaille, à plus forte raison ne devient-on pas capable de tirer un revenu de son sol sans avoir fait chèrement sa connaissance.

Lorsqu'il s'agit d'un étang, les rèqles générales aident moins encore que lorsqu'il s agit de labours on dherbages: les factemrs de réussite sont plus nombreux et plus cachés.

Nous ne prétendons donc pas éviter au jeune carpiculteur tous déboires : il doit faire ses écoles à ses frais.

Celte notion l'empêchera d ailleurs de se décourager si, son traité à la main, ayant appliqué toutes les règles de l’art, il constate une mésentente entre son auteur favori et la natune ironique. Car il y a de touchantes innocences. Il $m$ arrive de recevoir la visite, à Paris bien motendu, de propriétaires qui engagent ainsi le dialogue :

- Monsicur, j'ai quelques étangs et l'un m'a dit que volls me donneriez la manière d'obtenir vos résultats.

— Monsieur, lisez-vous le Bulletir de ITnion des Syndicnls de l'Elang?

- Majs je ne suis pas syndiqué !

- Syndiquez-vous, Monsieur, puis demander à votre président de visiter vos étangs. Il vous en apprendra plus en un jour, sur place, que moi en dix ans à Paris.

Fit voilà bien le premier conseil à donner : faites venir un homme d'expérience, connaissant votre pays. Traitez-le avec magnificence : faisans, canard, votre meilleur bourgogne rouge seront agréables à tout étagneux. Notez pas à pas ses remarques. Dressez des croquis. Faites-vous expliquer 
le maniement du matériel et des poissons. Véconomisez sur rien ce jourlà : ce sont des frais placés à $100 \%$.

Récapitulez la consultation le soir, avec votre hòte attendri par votre marc centenaire.

Néditez les jours suivants, en observant ros étangs.

Pronez votre bàton de pèlerin el allez voir traviller les quelques bons beveurs. Notez ce qui, dans leur installation et leurs méthodes, est applicable chez vous.

El essayez de bien faire. Répartissez vos frais sur trois anuées : elles vous sonl en tout cas nécessaires pour comprendre le caractère de vos fonds.

Fancardage, congrais, sélection sont la triple source de votre future réussite. Ces trois notions inséparables sont aussi la sioule certitude que nous vous puissions donner ici. los arrivées d'eau, vos grilles, vos viviers I hiver dépendent de trop de conditions naturelles, de possibilités, pour vous être enseignés par l'écrit.

Votre président de syndicat vous guidera. Vous apprendrez de lui mille honnes choses, qui ne vous profiteront qu'en les soumettant à l'expérience rhez vous.

Si je ne vous ai pas révélé de formule magique en ces lignes, au moins ne vous ai-je pas égaré, et c'est peut-itre de vous que nous entendrons un jour la solution dr problèmes que nous fitudions vainement depuis tant d'années.

\section{EXPLOITATION DES ÉTANGS}

\section{Par M. Philibert PANNetifr}

Secrelaire gineinal de lenion nationale des Picheurs aux filets.

Depuis rq22, éporque oì j’ai écrit ma brochure "De la Production Piscicole ", je n'ai pu, à mon regret, ćtant absorbé romplètement par d'autres occupations plus pressantes, écrire, mème dans Le Pêchear Professionnel, quoi que ce soil sur les élangs. J'ai, par contre, depuis ce moment, en faisant de nombreuses piches ot empoissonnements dans des résrions bien différentes an point de vue exploilation d'élangs, pu me rendre compte qu’il y avait encore une masse de propriétaires d'élangs hien ignorants des premières notions de pisciculture.

Mon exposé de rgme avait pour oricrine la campagne menée contre la peche aux filets fluviale et pour but une comparaison entre la production piscicole des étaners et celle du domaine fluvial de la france; ces deux éléments paraissant, à première vue, hien distincts alors qu'ils sont, en réalité, intimement liés l'un à l'autre; je me réserve de le démontrer ulterieurement.

L'exposé qui va suivre pour les lecteurs du Bulletin Français de Pisci- 